\section{What will 2012 bring for Canadian pharmacists?}

You MAY NOT HAVE THOUGHT MUCH ABOUT IT YET, BUT AS A "LEAPLING"1 I can tell you that 2012 is definitely a Leap Year. Yes, this is one of those years with an extra day on the calendar and a few superstitions attached. Some groups are encouraging people to take a risk to change their community for the better this year. ${ }^{2}$ Others are hoping the extra day will bring a measure of good fortune, and I'm optimistic that this will be the case for pharmacy practice.

Two of this issue's articles (see pages 17 and 30) highlight some very positive progress made recently in the expansion of Canadian pharmacists' scopes of practice. While there's still work to be done to obtain enabling legislation all across the country and to secure appropriate reimbursement for prescribing and medication management activities in most jurisdictions, ${ }^{3}$ there seems to be some consistent and forward momentum at the moment.

I'm encouraged by the comments of some pharmacists from Alberta who repeatedly mentioned the ability to better look after their patients as being "the best part of having additional prescribing authorization." 4 These pharmacists unquestionably see themselves as patient care practitioners. But while I expected some turf protection and skepticism from other health professionals when changes like these are realized, it is a bit disheartening to hear that other pharmacists can be among the barriers to implementation of these expanded practice skills. ${ }^{4}$

A somewhat different position is put forward in another article (see page 35), where the authors analyze responses to the 2010 Bill 16 professional allowance crisis in Ontario and ask whether patient care figured prominently enough in the discussion. As with many things, it seems we take 2 steps forward and 1 back.

I encourage you to read these articles, respond with your thoughts and experiences and maybe do what you can this year to use that extra day for the sake of your patients, while being appropriately reimbursed for your time and knowledge.

Find us on

Facebook

\section{Qu'apportera l'année 2012 aux pharmaciens canadiens?}

VOUS N'y AVEZ PEUT-ÊTRE PAS ENCORE BEAUCOUP PENSÉ, MAIS ÉTANT née un 29 février $^{1}$, je peux vous affirmer que 2012 est définitivement une année bissextile. Eh oui, c'est bel et bien l'une de ces années qui comptent un jour de plus et qui donnent lieu à quelques superstitions. Certains groupes encouragent les gens à prendre un risque dans le but d'améliorer leur communauté cette année ${ }^{2}$. D’autres espèrent que cette journée supplémentaire leur sera chanceuse, et je crois que ce sera le cas pour la pratique pharmaceutique.

Deux des articles de ce numéro (voir pages 17 et 30) présentent les progrès très positifs réalisés récemment en ce qui concerne l'expansion du champ d'activité des pharmaciens canadiens. Bien qu'il reste encore du travail à faire pour que des mesures législatives habilitantes soient en vigueur partout au pays et pour que les activités de prescription et de gestion des médicaments soient remboursées de façon appropriée dans la plupart des provinces ${ }^{3}$, il semble y avoir un élan indéniable en ce sens à l'heure actuelle.

Je suis encouragée par le fait que certains pharmaciens en Alberta ont mentionné à plusieurs reprises que le plus grand avantage des autorisations de prescription additionnelles était d'être en mesure de mieux prendre en charge leurs patients ${ }^{4}$. Ces pharmaciens se voient sans l'ombre d'un doute comme des professionnels de la santé. Je m'attendais à quelques réticences et à du scepticisme de la part des autres professionnels de la santé en raison de la nature des changements, mais il est un peu démoralisant d'entendre que d'autres pharmaciens peuvent faire partie des obstacles à l'expansion des compétences ${ }^{4}$.

Un point de vue quelque peu différent est présenté dans un autre article (voir page 35). Les auteurs analysent les réactions à la crise liée aux indemnités professionnelles du projet de loi 16 de 2010 en Ontario et se demandent si les soins aux patients ont été suffisamment pris en compte dans la discussion. Comme c'est souvent le cas, il semble que nous fassions deux pas en avant et un pas en arrière.

Je vous invite à lire ces articles, à nous faire part de vos commentaires et de vos expériences, et peut-être à faire ce que vous pouvez cette année pour le bien de vos patients dans le cadre de cette journée supplémentaire, tout en étant remboursé de façon appropriée pour votre temps et vos connaissances.

Courriel : rkilleen@pharmacists.ca,ou sur Facebook. 


\section{References}

1. Wikipedia. February 29. Available: http://en.wikipedia.org/wiki/February_29 (accessed November 30, 2011).

2. The Leap Year Project. Available: http://leapyearproject.org/ (accessed December 1, 2011).

3. Blueprint for Pharmacy. Cross-jurisdictional scan of legislative and policy framework. Available: www.blueprintforpharmacy.ca/docs/pdfs/2011/10/19/blueprintpolicy-framework---graphics---october-2011.pdf?Status=Master (accessed November 30, 2011).

4. Charrois T, Rosenthal M, Tsuyuki RT. Stories from the trenches: experiences of Alberta pharmacists in obtaining additional prescribing. Can Pharm J 2012;145:30-4. 